\title{
Bivariate Weibull Distributions Derived From Copula Functions In The Presence Of Cure Fraction And Censored Data
}

\author{
Em'1lio A. Coelho-Barros $^{1 *}$, Jorge Alberto Achcar ${ }^{2}$, Josmar Mazucheli $^{3}$ \\ ${ }^{1}$ Universidade Tecnol'ogica Federal do Paran'a, DAMAT/UTFPR \\ ${ }^{2}$ Universidade de $S^{\sim}$ ao Paulo, FMRP/USP \\ ${ }^{3}$ Universidade Estadual de Maring' $a$, DEs/UEM
}

\begin{abstract}
In this paper we introduce bivariate Weibull distributions derived from copula functions in presence of cure fraction, censored data and covariates. Two copula functions are explored: the FGM (Farlie - Gumbel Morgenstern) copula and the Gumbel copula. Inferences for the proposed models are obtained under the Bayesian approach, using standard MCMC (Markov Chain Monte Carlo) methods. An illustration of the proposed methodology is given considering a medical data set.
\end{abstract}

Key words: Bayesian methods, Bivariate Weibull, Censored observations, Copula functions, Cure fraction.

\section{Introduction}

A long-term survivor mixture model, also known as standard cure rate model, assumes that the studied population is a mixture of susceptible individuals, who experience the event of interest and non susceptible individuals that will never experience it. These individuals are not at risk with respect to the event of interest and are considered immune, non susceptible or cured Maller and Zhou (1996). Different approaches, parametric and non-parametric, have been considered to model the proportion of immunes and interested readers can refer, for example, to Boag (1949), Berkson (1952), Haybittle (1965), Farewell (1982, 1986), Meeker (1987), Dunsmuir et al., (1989), Taylor, (1995), Gamel et al. (1990, 1999), Ghitany and Maller (1992), Yamaguchi, (1992), Copas and Heydary (1997), Ng and McLachlan (1998), De Angelis et al. (1999), Peng and Dear (2000), Sy and Taylor (2000), Cancho and Bolfarine, (2001), Yu et al. (2004), Kannan et al., (2010).

Cure models for paired and clustered survival data have been considered by a number of authors, Wienke et al. $(2003,2006)$ introduced a model for a cure fraction in bivariate time-to-event data motivated by the article of Chatterjee and Shih (2001). Chatterjee and Shih (2003) used a correlated gamma frailty model by a copula function to analyse bivariate survival data.

\footnotetext{
${ }^{*}$ Corresponding author.
} 
Considering univariate lifetimes and following Maller and Zhou (1996), the standard cure rate model assumes that a certain fraction $p$ in the population is cured or never fail with respect to the specific cause of death or failure, while the remaining $(1-p)$ fraction of the individuals is not cured, leading to the survival function for the entire population written as,

$$
S(t)=p+(1-p) S_{0}(t)
$$

where $p \in(0,1)$ is the mixing parameter and $S_{0}(t)$ denotes a proper survival function for the non cured group in the population.

From the mixture survival function, (1), the probability density function is obtained from $f$ $(t)=-d_{t}-\frac{d}{-} S(t)$ and given by,

$$
f(t)=(1-p) f_{0}(t)
$$

where $f_{0}(t)$ is the probability density function for a susceptible individual. From $h(t)=\frac{f(t)}{S(t)}$, the hazard function is given by,

$$
h(t)=\frac{(1-p) f_{0}(t)}{p+(1-p) S_{0}(t)} .
$$

An alternative to a standard long-term survivor mixture model is the longterm survivor non-mixture model suggested by Yakovlev et al. (1996); Tsodikov et al. (2003); Lambert et al. (2010) which defines an asymptote for the cumulative hazard and hence for the cure fraction. The non-mixture model or the promotion time cure fraction has been used by Lambert et al. $(2007,2010)$ to estimate the probability of cure fraction in cancer lifetime data.

In this paper we consider a generalization of the mixture cure fraction model (1) for the bivariate case considering different copula functions to capture the possible existing dependence between two lifetimes $T_{1}$ and $T_{2}$ for the susceptible individuals, censored data and the presence of covariates. Inferences for the proposed models are obtained using Bayesian methods where samples of the joint posterior distribution of interest is simulated using MCMC (Markov Chain Monte Carlo) methods, as the popular Gibbs sampling algorithm Gelfand and Smith (1990); Casella and George (1992) and the Metropolis-Hastings algorithm Chib and Greenberg (1995).

The paper is organized as follows: in Section 2 we introduce bivariate lifetime data assuming mixture cure fraction models; in Section 3 we present the likelihood function considering bivariate lifetime data in presence of cure fraction and censored data considering two special copula functions: Farlie-GumbelMorgenstern (FGM) copula and Gumbel copula; in Section 4 we introduce the Weibull marginal distributions for the lifetimes $T_{1}$ and $T_{2}$; in Section 5 we introduce an illustrative example. Finally, in Section 6 we present some concluding remarks.

\section{Bivariate Lifetime Data Assuming Mixture Cure Fraction Models}

In some areas of application, especially in medical and engineering studies, we could have two lifetimes $T_{1}$ and $T_{2}$ associated with each individual or unit. Usually, these data are assumed to be independent, but in many cases, the lifetime of one component could affect the lifetime of 
the other component. This is the case as an example in the medical area of paired organs like kidneys, lungs, eyes, ears, dental implants among many others. In the literature we observe many papers related to bivariate lifetime parametric models Gumbel (1960); Freund (1961); Marshall and Olkin (1967); Downton (1970); Hawkes (1972); Block and Basu (1974); Hougaard (1986); Sarkar (1987); Arnold and Strauss (1988). An alternative is the use of copula functions Nelsen (2006); Trivedi and Zimmer (2005, 2006) to model bivariate lifetime data assuming different marginal lifetime distributions.

We also could have the presence of cure fraction or immunes for both lifetimes $T_{1}$ and $T_{2}$. In this way, let us assume mixture models for $T_{1}$ and $T_{2}$, given, respectively, by,

$$
\begin{aligned}
& S_{1}\left(t_{1}\right)=p_{1}+\left(1-p_{1}\right) S_{10}\left(t_{1}\right) \\
& S_{2}\left(t_{2}\right)=p_{2}+\left(1-p_{2}\right) S_{01}\left(t_{2}\right)
\end{aligned}
$$

where $S_{10}\left(t_{1}\right)$ and $S_{01}\left(t_{2}\right)$ are the survival functions for the susceptible individuals in the lifetimes $T_{1}$ and $T_{2}$, respectively; $p_{1}$ and $p_{2}$ are, respectively, the cure probabilities in $T_{1}$ and $T_{2}$; $\left(1-p_{1}\right)$ and $\left(1-p_{2}\right)$ are, respectively, the probabilities of susceptible in lifetimes $T_{1}$ and $T_{2}$.

The joint survival function for $T_{1}$ and $T_{2}$ is given by,

$$
S\left(t_{1}, t_{2}\right)=P\left(T_{1}>t_{1}, T_{2}>t_{2}\right)=1-F_{1}\left(t_{1}\right)-F_{2}\left(t_{2}\right)+F\left(t_{1}, t_{2}\right),
$$

where $F_{1}\left(t_{1}\right)=P\left(T_{1} \leq t_{1}\right)$ is the marginal cumulative distribution function for $T_{1} ; F_{2}\left(t_{2}\right)=P\left(T_{2}\right.$ $\left.\leq t_{2}\right)$ is the marginal cumulative distribution function for $T_{2}$;

$F\left(t_{1}, t_{2}\right)=P\left(T_{1} \leq t_{1}, T_{2} \leq t_{2}\right)$ is the joint cumulative distribution function for $T_{1}$ and $T_{2}$.

In this way, we get from (4),

$$
\begin{aligned}
& F_{1}\left(t_{1}\right)=1-S_{1}\left(t_{1}\right)=1-p_{1}-\left(1-p_{1}\right) S_{10}\left(t_{1}\right) ; \\
& F_{2}\left(t_{2}\right)=1-S_{2}\left(t_{2}\right)=1-p_{2}-\left(1-p_{2}\right) S_{01}\left(t_{2}\right) .
\end{aligned}
$$

Thus, from (5), we get,

$$
S\left(t_{1}, t_{2}\right)=p_{1}+p_{2}-1+\left(1-p_{1}\right) S_{10}\left(t_{1}\right)+\left(1-p_{2}\right) S_{01}\left(t_{2}\right)+F\left(t_{1}, t_{2}\right) .
$$

Let us define the binary indicator random variables $V_{1}$ and $V_{2}$ where $V_{1}=1$ if the individual is susceptible for the lifetime $T_{1}$ and $V_{1}=0$ if the individual is immune; in the same way, $V_{2}=1$ if the individual is susceptible for the lifetime $T_{2}$ and $V_{2}=0$ if the individual is immune, thus $P$ $\left(V_{1}=1\right)=1-p_{1}, P\left(V_{1}=0\right)=p_{1}, P\left(V_{2}=1\right)=1-p_{2}$ and $P\left(V_{2}=0\right)=p_{2}$.

Let us assume $V_{1}$ independent of $V_{2}$, that is,

$$
P\left(V_{1}=v_{1}, V_{2}=v_{2}\right)=P\left(V_{1}=v_{1}\right) P\left(V_{2}=v_{2}\right),
$$

where $v_{j}=1$ or 0 for $j=1,2$. That is, 
where $\varphi 00+\varphi 10+\varphi 01+\varphi 11=1$.

$$
\begin{gathered}
\varphi_{00}=\quad P\left(V_{1}=0, V_{2}=0\right)=p_{1} p_{2} ; \\
\varphi_{10}=\quad P\left(V_{1}=1, V_{2}=0\right)=\left(1-p_{1}\right) p_{2} ; \\
\varphi_{01}=\quad P\left(V_{1}=0, V_{2}=1\right)=p_{1}\left(1-p_{2}\right) ; \\
\varphi_{11}=\quad P\left(V_{1}=1, V_{2}=1\right)=\left(1-p_{1}\right)\left(1-p_{2}\right) ;
\end{gathered}
$$

Assuming $T_{1}$ independent of $T_{2}$, we have, $S\left(t_{1}, t_{2}\right)=S_{1}\left(t_{1}\right) S_{2}\left(t_{2}\right)$; from (4), we have,

$$
\begin{array}{ll}
S\left(t_{1}, t_{2}\right)=\quad & p_{1} p_{2}+\left(1-p_{1}\right) p_{2} S_{10}\left(t_{1}\right)+p_{1}\left(1-p_{2}\right) S_{01}\left(t_{2}\right)+ \\
& \left(1-p_{1}\right)\left(1-p_{2}\right) S_{10}\left(t_{1}\right) S_{01}\left(t_{2}\right),
\end{array}
$$

assuming $T_{1}$ dependent of $T_{2}$, we get from (9),

$$
S(t 1, t 2)=\varphi 00+\varphi 10 S 10(t 1)+\varphi 01 S 01(t 2)+\varphi 11 S 110(t 1, t 2)
$$

where $S_{110}\left(t_{1}, t_{2}\right)$ is the joint survival function for $T_{1}$ and $T_{2}$ for the susceptible individuals.

Different bivariate lifetime distributions could be used for $S_{110}\left(t_{1}, t_{2}\right)$ Block and Basu (1974); Marshall and Olkin (1967); Sarkar (1987). Another possibility is the use of copula functions Nelsen (2006). Copula functions can be used to link marginal distributions with a joint distribution. For specified univariate marginal distribution functions $F_{1}\left(t_{1}\right), F_{2}\left(t_{2}\right), \ldots, F_{m}\left(t_{m}\right)$, the function,

$$
C\left(F_{1}\left(t_{1}\right), F_{2}\left(t_{2}\right), \ldots, F_{m}\left(t_{m}\right)\right)=F\left(t_{1}, t_{2}, \ldots, t_{m}\right),
$$

which is defined using a copula function $C$, results in a multivariate distribution function with univariate distributions specified as $F_{1}\left(t_{1}\right), F_{2}\left(t_{2}\right), \ldots, F_{m}\left(t_{m}\right)$.

It is important to point out that any multivariate distribution function $F$ can be written in the form of a copula function Sklar (2010), that is, if $F\left(t_{1}, t_{2}, \ldots, t_{m}\right)$ is a joint multivariate distribution function with univariate marginal distribution functions $F_{1}\left(t_{1}\right), F_{2}\left(t_{2}\right), \ldots, F_{m}\left(t_{m}\right)$, thus there exist a copula function $C\left(u_{1}, u_{2}, \ldots, u_{m}\right)$, such that,

$$
F\left(t_{1}, t_{2}, \ldots, t_{m}\right)=C\left(F_{1}\left(t_{1}\right), F_{2}\left(t_{2}\right), \ldots, F_{m}\left(t_{m}\right)\right)
$$

If every $F_{l}$ is continuous, then $C$ is unique. For the special case of bivariate distributions, we have $m=2$.

The approach to formulate a multivariate distribution using a copula is based on the idea that a simple transformation can be made of each marginal variable in such a way that each transformed marginal variable has a uniform distribution. Once this is done, the dependence structure can be expressed as a multivariate distribution on the obtained uniforms, and a copula 
is precisely a multivariate distribution on marginally uniform random variables. In this way, there are many families of copulas which differ in the detail of the dependence they represent. In the bivariate case, let $T_{1}$ and $T_{2}$ be two random variables with continuous distribution functions $F_{1}$ and $F_{2}$.

The probability integral transform can be applied separately to the two random variables to define $U=F_{1}\left(t_{1}\right)$ and $V=F_{2}\left(t_{2}\right)$, where $U$ and $V$ have uniform $(0,1)$ distributions, but are usually dependent if $T_{1}$ and $T_{2}$ are dependent ( $T_{1}$ and $T_{2}$ independent, implies that $U$ and $V$ are independent). Specifying dependence between $T_{1}$ and $T_{2}$ is the same as specifying dependence between $U$ and $V$. With $U$ and $V$ uniform random variables, the problem reduces to specifying a bivariate distribution between two uniforms, that is, a copula.

As a first copula function appropriated to model weak dependences, we consider a FarlieGumbel-Morgenstern (FGM) copula Morgenstern (1956), given by,

$$
F 110(t 1, t 2)=F 10(t 1) F 01(t 2)[1+\theta 1 S 10(t 1) S 01(t 2)]
$$

where the dependence parameter $\theta_{1}$ is defined in the interval $[-1,1]$.

From (5), the joint survival function $S_{110}\left(t_{1}, t_{2}\right)$ for $T_{1}$ and $T_{2}$ considering the susceptible individuals is given by,

$$
S 110(t 1, t 2)=1-F 10(t 1)-F 01(t 2)+F 110(t 1, t 2),
$$

that is, for the susceptible individuals,

$$
\mathrm{S} 110(\mathrm{t} 1, \mathrm{t} 2)=\mathrm{S} 10(\mathrm{t} 1) \mathrm{S} 01(\mathrm{t} 2)[1+\theta 1 \mathrm{~F} 10(\mathrm{t} 1) \mathrm{F} 01(\mathrm{t} 2)]
$$

From (11) we get,

$$
S(t 1, t 2)=\varphi 00+\varphi 10 S 10(t 1)+\varphi 01 S 01(t 2)+\varphi 11 A(t 1, t 2),
$$

where $A\left(t_{1}, t_{2}\right)=S_{10}\left(t_{1}\right) S_{01}\left(t_{2}\right)\left[1+\theta_{1} F_{10}\left(t_{1}\right) F_{01}\left(t_{2}\right)\right]$.

As a second copula function, we assume the Gumbel copula Gumbel (1960) given by,

$$
\begin{aligned}
F 110(t 1, t 2)=\quad & F 10(t 1)+F 01(t 2)-1+S 10(t 1) S 01(t 2) \times \\
& \exp \left\{-\theta_{2} \log \left[S_{10}\left(t_{1}\right)\right] \log \left[S_{01}\left(t_{2}\right)\right]\right\},
\end{aligned}
$$

where $0 \leq \theta_{2} \leq 1$.

That is, from (15), we get,

$$
S_{110}\left(t_{1}, t_{2}\right)=S_{10}\left(t_{1}\right) S_{01}\left(t_{2}\right) \exp \left\{-\theta_{2} \log \left[S_{10}\left(t_{1}\right)\right] \log \left[S_{01}\left(t_{2}\right)\right]\right\} .
$$

From (11) we get, 


$$
S(t 1, t 2)=\varphi 00+\varphi 10 S 10(t 1)+\varphi 01 S 01(t 2)+\varphi 11 S 10(t 1) S 01(t 2) B(t 1, t 2)
$$

where $B\left(t_{1}, t_{2}\right)=\exp \left\{-\theta_{2} \log \left[S_{10}\left(t_{1}\right)\right] \log \left[S_{01}\left(t_{2}\right)\right]\right\}$.

\section{Bivariate Lifetime Data In Presence Of Cure Fraction And Censored Data}

Let us assume $n$ pairs of a random sample of lifetimes $t_{1 i}$ and $t_{2 i}, i=1, \ldots, n$, that can be right censored where censoring is independent of the lifetimes divided into four classes:

$C_{1}$ : both lifetimes $t_{1 i}$ and $t_{2 i}$ are observed;

$C_{2}: t_{1 i}$ is a lifetime and $t_{2 i}$ is a censored time (that is, we only know that $T 2 i \geq t 2 i$ );

$C_{3}: t_{1 i}$ is a censoring time and $t_{2 i}$ is a lifetime (that is, we only know that $T 1 i \geq t 1 i$ );

$C_{4}$ : both $t_{1 i}$ and $t_{2 i}$ are censoring times.

It is important to note that we could have other censoring schemes but this will be not considered in this paper.

The contribution of the $i^{t h}$ individual for the likelihood function Lawless (1982) is given by,

$$
L=\prod_{i \in C_{1}} f\left(t_{1 i}, t_{2 i}\right) \prod_{i \in C_{2}}\left[-\frac{\partial S\left(t_{1 i}, t_{2 i}\right)}{\partial t_{1 i}}\right] \prod_{i \in C_{3}}\left[-\frac{\partial S\left(t_{1 i}, t_{2 i}\right)}{\partial t_{2 i}}\right] \prod_{i \in C_{4}} S\left(t_{1 i}, t_{2 i}\right)
$$

where $f\left(t_{1 i}, t_{2 i}\right)$ is the joint probability density function for $T_{1}$ and $T_{2} ; S\left(t_{1 i}, t_{2 i}\right)$ is the joint survival function; $\frac{\partial S\left(t_{1 i}, t_{2 i}\right)}{\partial t_{1 i}}$ and $\frac{\partial S\left(t_{1 i}, t_{2 i}\right)}{\partial t_{2 i}}$ are the partial derivatives of $S\left(t_{1 i}, t_{2 i}\right)$, with respect to $t_{1 i}$ and $t_{2 i}$, respectively.

Let us define the indicator variables $\delta_{1 i}$ and $\delta_{2 i}$, by,

1 if $t_{j i}$ is an observed lifetime

0 if $t_{j i}$ is a censured lifetime

for $j=1,2 ; i=1,2, \ldots, n$.

In this way, we rewrite the likelihood function (21) as,

$$
\begin{aligned}
L= & \prod_{i=1}^{n}\left[f\left(t_{1 i}, t_{2 i}\right)\right]^{\delta_{1 i} \delta_{2 i}} \prod_{i=1}^{n}\left[-\frac{\partial S\left(t_{1 i}, t_{2 i}\right)}{\partial t_{1 i}}\right]^{\delta_{1 i}\left(1-\delta_{2 i}\right)} \times \\
& \prod_{i=1}^{n}\left[-\frac{\partial S\left(t_{1 i}, t_{2 i}\right)}{\partial t_{2 i}}\right]^{\left(1-\delta_{1 i}\right) \delta_{2 i}} \prod_{i=1}^{n}\left[S\left(t_{1 i}, t_{2 i}\right)\right]^{\left(1-\delta_{1 i}\right)\left(1-\delta_{2 i}\right)}
\end{aligned}
$$




\subsection{Farlie-Gumbel-Morgenstern Copula}

From (17), we get expressions for $f\left(t_{1}, t_{2}\right),-\frac{\partial S\left(t_{1}, t_{2}\right)}{\partial t_{1}},-\frac{\partial S\left(t_{1}, t_{2}\right)}{\partial t_{2}}$ and $S\left(t_{1}, t_{2}\right)$ needed in the likelihood function (23), given by:

$$
f\left(t_{1}, t_{2}\right)=\frac{\partial^{2} F\left(t_{1 i}, t_{2 i}\right)}{\partial t_{1 i} \partial t_{2 i}}=-\frac{\partial^{2} S\left(t_{1 i}, t_{2 i}\right)}{\partial t_{1 i} \partial t_{2 i}}
$$

1. $1+S\left(t_{1 i}, t_{2 i}\right)$. , where $F\left(t_{1 i}, t_{2 i}\right)=F_{1}\left(t_{1}\right)+F_{2}\left(t_{2}\right)-$

From (17), we get,

$$
\begin{aligned}
& \frac{\partial A\left(t_{1}, t_{2}\right)}{\partial t_{1}}=-f_{10}\left(t_{1}\right) S_{01}\left(t_{2}\right)+\theta_{1} S_{01}\left(t_{2}\right) F_{01}\left(t_{2}\right)\left[-f_{10}\left(t_{1}\right) F_{10}\left(t_{1}\right)+f_{10}\left(t_{1}\right) S_{10}\left(t_{1}\right)\right] \\
& \text { and } \\
& \frac{\partial A\left(t_{1}, t_{2}\right)}{\partial t_{1} t_{2}}=f_{10}\left(t_{1}\right) S_{01}\left(t_{2}\right)+\theta_{1} S_{01}\left(t_{2}\right) F_{01}\left(t_{2}\right) f_{10}\left(t_{1}\right)\left[1-2 F_{10}\left(t_{1}\right)\right] .
\end{aligned}
$$

From the expression (25), we get,

$$
f\left(t_{1}, t_{2}\right)=\varphi_{11} f_{10}\left(t_{1}\right) f_{01}\left(t_{2}\right)\left\{1+\theta_{1}\left[1-2 F_{10}\left(t_{1}\right)\right]\left[1-2 F_{01}\left(t_{2}\right)\right]\right\} .
$$

2. From (17), we get,

$$
-\frac{\partial S\left(t_{1}, t_{2}\right)}{\partial t_{1}}=f_{10}\left(t_{1}\right)\left\{\phi_{10}+\phi_{11} S_{01}\left(t_{2}\right)-\phi_{11} \theta_{1} S_{01}\left(t_{2}\right) F_{01}\left(t_{2}\right)\left[1-2 F_{10}\left(t_{1}\right)\right]\right\}
$$

3. From (17), we get,

$$
-\frac{\partial S\left(t_{1}, t_{2}\right)}{\partial t_{2}}=f_{01}\left(t_{2}\right)\left\{\phi_{01}+\phi_{11} S_{10}\left(t_{1}\right)-\phi_{11} \theta_{1} S_{10}\left(t_{1}\right) F_{10}\left(t_{1}\right)\left[1-2 F_{01}\left(t_{2}\right)\right]\right\}
$$

4. Observe that $S\left(t_{1}, t_{2}\right)$ needed in the likelihood function (23) is given by (17).

\subsection{Gumbel Copula}

From (20), we get,

$$
\begin{aligned}
& \frac{\partial B\left(t_{1}, t_{2}\right)}{\partial t_{1}}=\frac{\theta_{2} f_{10}\left(t_{1}\right) \log \left[S_{01}\left(t_{2}\right)\right] B\left(t_{1}, t_{2}\right)}{S_{10}\left(t_{1}\right)} \\
& \frac{\partial B\left(t_{1}, t_{2}\right)}{\partial t_{2}}=\frac{\theta_{2} f_{01}\left(t_{2}\right) \log \left[S_{10}\left(t_{1}\right)\right] B\left(t_{1}, t_{2}\right)}{S_{01}\left(t_{2}\right)}
\end{aligned}
$$

and, 


$$
\begin{aligned}
& \frac{\partial S\left(t_{1}, t_{2}\right)}{\partial t_{1}}=-\phi_{10} f_{10}\left(t_{1}\right)+\phi_{11} S_{01}\left(t_{2}\right)\left[-f_{10}\left(t_{1}\right) B\left(t_{1}, t_{2}\right)+S_{10}\left(t_{1}\right) \frac{\partial B\left(t_{1}, t_{2}\right)}{\partial t_{1}}\right] ; \\
& \frac{\partial S\left(t_{1}, t_{2}\right)}{\partial t_{2}}=-\phi_{10} f_{01}\left(t_{2}\right)+\phi_{11} S_{10}\left(t_{1}\right)\left[-f_{01}\left(t_{2}\right) B\left(t_{1}, t_{2}\right)+S_{01}\left(t_{2}\right) \frac{\partial B\left(t_{1}, t_{2}\right)}{\partial t_{2}}\right] .
\end{aligned}
$$

In this way, we get,

$$
\begin{gathered}
f\left(t_{1}, t_{2}\right)=\quad \varphi_{11} f_{10}\left(t_{1}\right) f_{01}\left(t_{2}\right) B\left(t_{1}, t_{2}\right)\left\{1-\theta_{2}-\theta_{2} \log \left[S_{10}\left(t_{1}\right)\right]-\right. \\
\left.\theta_{2} \log \left[S_{01}\left(t_{2}\right)\right]+\theta_{2}^{2} \log \left[S_{10}\left(t_{1}\right)\right] \log \left[S_{01}\left(t_{2}\right)\right]\right\} \\
-\frac{\partial S\left(t_{1}, t_{2}\right)}{\partial t_{1}}=f_{10}\left(t_{1}\right)\left\{\phi_{10}+\phi_{11} S_{01}\left(t_{2}\right)\left[1-\theta_{2} \log \left[S_{01}\left(t_{2}\right)\right] B\left(t_{1}, t_{2}\right)\right]\right\} \\
-\frac{\partial S\left(t_{1}, t_{2}\right)}{\partial t_{2}}=f_{01}\left(t_{2}\right)\left\{\phi_{01}+\phi_{11} S_{10}\left(t_{1}\right)\left[1-\theta_{2} \log \left[S_{10}\left(t_{1}\right)\right] B\left(t_{1}, t_{2}\right)\right]\right\}
\end{gathered}
$$

where $B\left(t_{1}, t_{2}\right)$ is defined in $(20)$.

Different marginal parametric lifetime distributions could be used for the lifetimes $T_{1}$ and $T_{2}$, as exponential, Weibull, log-normal, gamma or generalized gamma distributions.

\section{Weibull Marginal Distributions For The Lifetimes $T_{1}$ And $T_{2}$}

Considering the bivariate lifetime distributions in presence of cure fractions derived from mixture models with a dependence structure given by FGM or Gumbel copula functions, we assume as a special model, a popular lifetime model extensively used in medical or engineering applications: the Weibull distribution. In this way, we consider marginal Weibull distributions for the lifetimes $T_{1}$ and $T_{2}$ of susceptible individuals with densities and survival functions given, respectively, by,

$$
\begin{aligned}
f_{10}\left(t_{1}\right) & =\frac{\lambda_{1}}{\mu_{1}}\left(\frac{t_{1}}{\mu_{1}}\right)^{\lambda_{1}-1} \exp \left[-\left(\frac{t_{1}}{\mu_{1}}\right)^{\lambda_{1}}\right] ; \\
f_{01}\left(t_{2}\right)= & \frac{\lambda_{2}}{\mu_{2}}\left(\frac{t_{2}}{\mu_{2}}\right)^{\lambda_{2}-1} \exp \left[-\left(\frac{t_{2}}{\mu_{2}}\right)^{\lambda_{2}}\right] ; \\
S_{10}\left(t_{1}\right) & =\exp \left[-\left(\frac{t_{1}}{\mu_{1}}\right)^{\lambda_{1}}\right] ; \\
S_{01}\left(t_{2}\right) & =\exp \left[-\left(\frac{t_{2}}{\mu_{2}}\right)^{\lambda_{2}}\right] .
\end{aligned}
$$


The Weibull distribution is very popular to analyse lifetime data, since its hazard function $h(t)=f(t) / S(t)$ is increasing if the shape parameter $\lambda>1$; is decreasing if $\lambda<1$ or is constant (an exponential distribution) if $\lambda=1$ Lawless (1982).

From (2), the means for lifetimes $T_{1}$ and $T_{2}$ are given respectively, by,

$$
\begin{aligned}
& E\left(T_{1}\right)=\left(1-p_{1}\right) \mu_{1} \Gamma\left[1+\frac{1}{\lambda_{1}}\right] ; \\
& E\left(T_{2}\right)=\left(1-p_{2}\right) \mu_{2} \Gamma\left[1+\frac{1}{\lambda_{2}}\right],
\end{aligned}
$$

where $p 1=\varphi 00+\varphi 01$ and $p 2=\varphi 00+\varphi 10$.

We get inferences for the vector parameter $\phi_{\mathbf{r}}=\left(\lambda_{1}, \lambda_{2}, \mu_{1}, \mu_{2}, \varphi_{00}, \varphi_{10}, \varphi_{01}, \varphi_{11}, \theta_{r}\right)$;

where $r=1$ (FGM copula), $r=2$ (Gumbel copula), $\varphi_{00}+\varphi_{10}+\varphi_{01}+\varphi_{11}=1, \lambda_{1}$ and $\lambda_{2}$ are shape parameters, $\mu_{1}$ and $\mu_{2}$ are scale parameters for the marginal Weibull distributions for $T_{1}$ and $T_{2}$ using Bayesian methods where samples of the joint posterior distribution of interest are simulated using MCMC (Markov Chain Monte Carlo) methods, as the popular Gibbs sampling algorithm Gelfand and Smith (1990); Casella and George (1992) and the Metropolis-Hastings algorithm. Chib and Greenberg (1995). Assuming prior independence among the parameters, we consider uniform or gamma prior distributions for the parameters $\lambda_{1}, \lambda_{2}, \mu_{1}, \mu_{2}$ and $\theta_{r}, r=1,2$.

A Dirichlet prior distribution is considered for the incidence parameters $\varphi_{11}, \varphi_{10}, \varphi_{01}$, and $\varphi_{00}$, where $\varphi_{11}+\varphi_{10}+\varphi_{01}+\varphi_{00}=1$, with density generically given by,

$$
f\left(x_{1}, \ldots, x_{k-1} ; w_{1}, \ldots, w_{k}\right)=\frac{1}{B(\mathbf{w})} \prod_{i=1}^{k} x_{i}^{w_{i}-1},
$$

for all $x_{1}, \ldots, x_{k-1}>0$ satisfying $x_{1}+\cdots+x_{k-1}<1$, where $x_{k}$ is given by $1-x_{1}-\cdots-x_{k-1}$. The normalizing constant is the multinomial beta function, which can be expressed in terms of the gamma function:

$$
B(\mathbf{w})=\frac{\prod_{i=1}^{k} \Gamma\left(w_{i}\right)}{\Gamma\left(\sum_{i=1}^{k} w_{i}\right)}
$$

In the presence of covariates associated to each bivariate lifetime $T_{1}$ and $T_{2}$, we could assume the following regression model,

$$
\begin{aligned}
& \mu_{1 i}=\alpha_{1} \exp \left({ }_{1}^{\prime} \mathbf{x}_{\mathbf{i}}\right) ; \\
& \mu_{2 i}=\alpha_{2} \exp \left({ }_{2}^{\prime} \mathbf{x}_{\mathbf{i}}\right) ;
\end{aligned}
$$

where $\mathbf{x}_{\mathbf{i}}=\left(x_{1 i}, \ldots, x_{k i}\right)^{0}$ is a vector of covariates and $\beta_{\mathbf{l}}=\left(\beta_{l 1}, \ldots, \beta_{l k}\right), l=1,2$ denotes a vector regression parameters.

We also assume Normal distributions for the regression coefficients $\beta_{l s}$, and Gamma prior distributions for $\alpha_{l}, l=1,2 ; s=1, \ldots, k$.

\section{An Example}


In this application, we consider a data set Huster et al. (1989) with 197 patients were a 50\% random sample of the patients with "high-risk" diabetic retinopathy as defined by the Diabetic Retinopathy Study (DRS). Each patient had one eye randomized to laser treatment and the other eye received no treatment. For each eye, the event of interest was the time from initiation of treatment to the time when visual acuity dropped below 5/200 two visits in a row (call it "blindness"). Thus there is a built-in lag time of approximately 6 months (visits were every 3 months). Survival times in this data set are therefore the actual time to blindness in months, minus the minimum possible time to event (6.5 months). Censoring was caused by death, dropout, or end of the study. This data set can be obtained in http://www.mayo.edu/research/documents/diabeteshtml/DOC-10027460

In the analysis considered here, we assume as lifetimes, the times (in months) to blindness for the eye randomized to laser treatment $\left(T_{1}\right)$, with 143 censored observations and 54 notcensored observations, and the time (in months) to blindness for the eye randomized that not received the treatment $\left(T_{2}\right)$, with 96 censored observations and 101 not-censored observations. Two covariates, the age at diagnosis of diabetes (in years) and the type of diabetes (juvenile, adult) were considered. Observe that among the censored observations, some patients will never have the occurrence of the event of interest, that is, they are immunes.

As a preliminary analysis of the data, we present in Figure 1, the KaplanMeier nonparametric estimates for the survival functions considering the two lifetimes associated to each patient (treated and untreated eyes). From these plots, we have some indication of cure fraction (a large number of censoring times at the end of study). From the Kaplan-Meier plots of Figure 1, we also observe better results for the treated eyes, that is, larger times to blindness in comparison with the untreated eyes. From the Kaplan-Meier estimates we have estimates for the mean time to blindness: 57.83 months for the treated eyes and 43.53 months for the untreated eyes.

In medical research, it is common to assume independence between $T_{1}$ and $T_{2}$, which could be not appropriated.

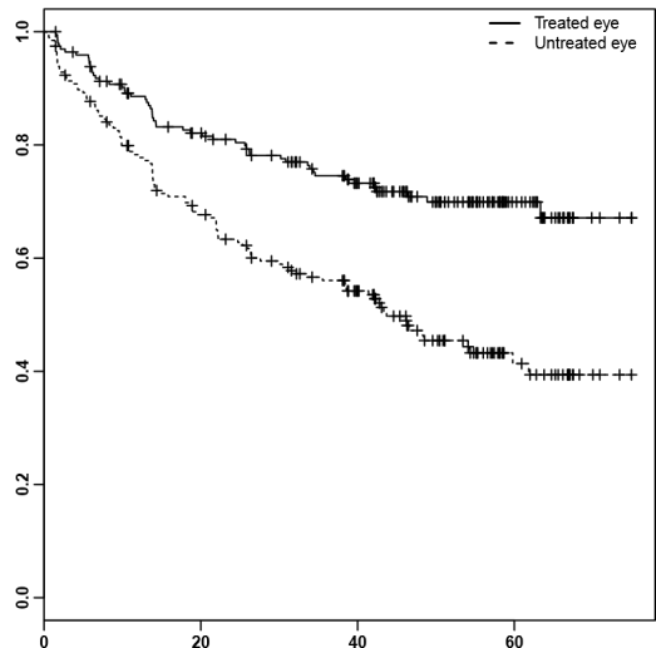

Figure 1: Kaplan-Meier nonparametric estimates for the survival function (0:censored; 1:blindness). 
As a first model, we assume a bivariate Weibull lifetime distribution derived from the FGM copula in presence of cure fraction presented in Section 3.1 with independent priors $\lambda_{l} \sim$ $\operatorname{Gamma}(0.001,0.001), \mu_{l} \sim \operatorname{Gamma}(0.001,0.001), l=1,2$, and $\theta_{1} \sim U(-1,1)$, were $\operatorname{Gamma}(a, b)$ denotes a gamma distribution with mean $a / b$ and variance $a / b^{2}$ and $U(c, d)$ denotes an uniform distribution defined in the interval $(c, d)$. We also assume a Dirichlet prior given by (38) for $\varphi_{11}$, $\varphi_{10}, \varphi_{01}$ and $\varphi_{00}$ with hyperparameter values $w_{1}=w_{2}=w_{3}=w_{4}=1$ (noninformative priors for the incidence parameters).

Considering the rjags library Plummer (2011) in R software R Development Core Team (2011), a single chain has been used considering the simulation for each parameter for 200,000 times with a burn sample size of 50,000 to eliminate the possible effect of the initial values. We choose the simulated values from 100 to 100 , to get approximated uncorrelated values which result in a final chain of size 2,000 . We automatically generate initial values by sampling from the prior for the parameters, since the priors are not totally diffuse (vague). Convergence of the Gibbs Sampling algorithm was monitored using standard graphical methods, as the trace plots and the estimated posterior densities of the simulated samples Brooks and Gelman (1998). In Figure 2 we have an example considering the parameter $\lambda_{1}$ from the FGM copula (first fitted model), all the other parameters have similar behavior. Note we did not show the full conditional distributions since we consider the rjags library, making it unnecessary to use, however in appendix A we show the required codes to obtain the Bayesian estimates.
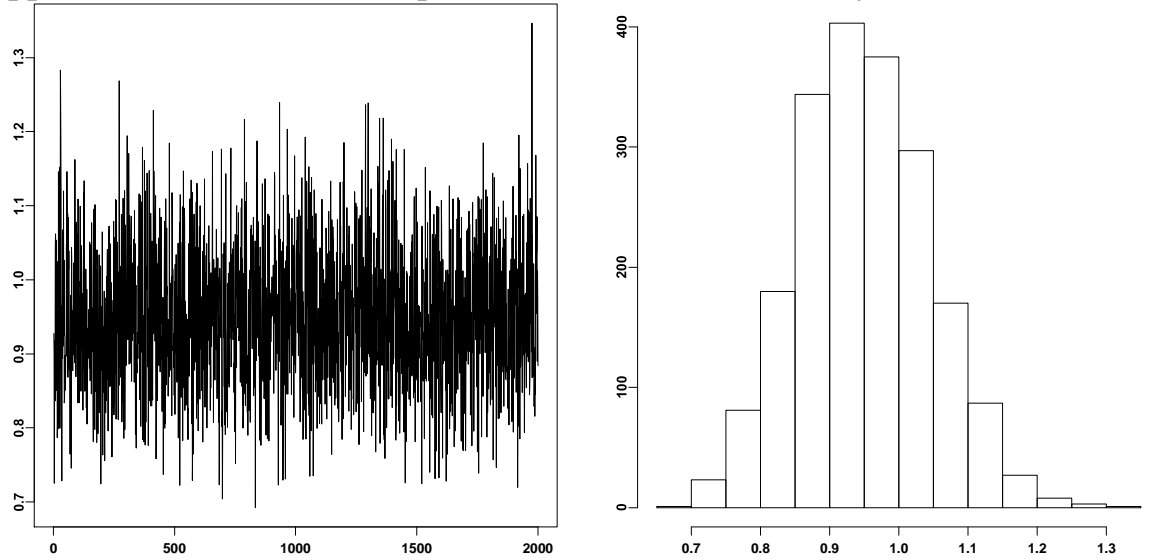

Figure 2: Trace plot and estimated posterior density of the simulated samples considering the parameter $\lambda_{1}$ from the FGM copula.

In Table 1, we have the posterior summaries of interest (Monte Carlo estimates of the posterior means for each parameter). We also have in Table 1, the Monte Carlo estimate of DIC (Deviance Information Criterion), introduced by Spiegelhalter et al. (2002), used as a discrimination criterion for different models. Smaller values of DIC indicates better models. The deviance can be expressed as,

$$
D(\theta)=-2 \log L(\theta \mid \mathbf{y})+c
$$


where $L(\theta \mid \mathbf{y})$ is the likelihood function for the unknown parameters in $\theta$ given the observed data $\mathbf{y}$ and $c$ is a constant not required for comparing models. Spiegelhalter et al. (2002) defined the DIC criterion by,

$$
D I C=D(\theta)+2 n_{D},
$$

where $D\left(\theta^{\wedge}\right)$ is the deviance evaluated at the posterior mean $\theta^{\wedge}$ and $n_{D}$ is the effective number of parameters in the model, namely $n_{D}=D^{-}-D\left(\theta^{\wedge}\right)$, where $D^{-}=E[D(\theta)]$ is the posterior deviance measuring the quality of the goodness-offit of the current model to the data.

Table 1: Posterior summaries (bivariate Weibull distribution derived from FGM copula in presence of cure fraction)

\begin{tabular}{ccccc}
\hline Parameter & Mean & S.D. & $95 \%$ Credible Interval & DIC \\
\hline$\lambda_{1}$ & 0.93 & 0.15 & $(0.68 ; 1.26)$ & \\
$\lambda_{2}$ & 0.94 & 0.09 & $(0.77 ; 1.13)$ & \\
$\mu_{1}$ & 66.65 & 40.51 & $(22.88 ; 164.73)$ & \\
$\mu_{2}$ & 35.56 & 8.99 & $(22.88 ; 57.49)$ & \\
$\theta_{1}$ & 0.62 & 0.30 & $(-0.11 ; 0.98)$ & 1667.04 \\
$\varphi 11$ & 0.45 & 0.13 & $(0.25 ; 0.73)$ & \\
$\varphi 10$ & 0.08 & 0.05 & $(0.004 ; 0.20)$ & \\
$\varphi 01$ & 0.25 & 0.12 & $(0.02 ; 0.47)$ & \\
$\varphi 00$ & 0.22 & 0.07 & $(0.05 ; 0.35)$ & \\
& & & & \\
\hline
\end{tabular}

In Table 2, we have the posterior summaries of interest assuming independent marginal Weibull distributions for the lifetimes $T_{1}$ and $T_{2}$ in presence of cure fraction, that is, with $\theta_{1}=0$, assuming the same priors for the parameters of interest and the same Gibbs sampling steps using the rjags library in $\mathrm{R}$ software used for the bivariate Weibull distribution derived from FGM copula in presence of cure fraction (results in Table 1).

From the obtained Monte Carlo estimates for DIC assuming the two models given in Tables 1 and 2 (presence or not of dependence between $T_{1}$ and $T_{2}$ ) we observe that the bivariate Weibull distribution derived from a FGM copula function gives better fit for the data, since the obtained DIC value is smaller for the dependent model than for the independent model.

Table 2: Posterior summaries (independent Weibull distributions in presence of cure fraction)

\begin{tabular}{ccccc}
\hline Parameter & Mean & S.D. & 95\% Credible Interval & DIC \\
\hline$\lambda_{1}$ & 0.98 & 0.17 & $(0.71 ; 1.34)$ &
\end{tabular}




$\begin{array}{lcccc}\lambda_{2} & 0.95 & 0.09 & (0.78 ; 1.14) & \\ \mu_{1} & 49.52 & 31.28 & (20.33 ; 138.20) & \\ \mu_{2} & 33.97 & 7.18 & (23.18 ; 50.79) & \\ \varphi 11 & 0.39 & 0.12 & (0.24 ; 0.68) & 1669.76 \\ \varphi 10 & 0.05 & 0.04 & (0.003 ; 0.16) & \\ \varphi 01 & 0.29 & 0.12 & (0.02 ; 0.48) \\ \varphi 00 & 0.26 & 0.06 & (0.13 ; 0.36)\end{array}$

Considering "FGM copula model" in the presence of cure fraction and the covariates age and type of diabetes (see (40)), that is,

$$
\begin{gathered}
\mu 1 i=\alpha 1 \exp \left(\beta 11 x_{1 i}+\beta 12 x 2 i\right) \\
\mu 2 i=\alpha 2 \exp \left(\beta 21 x_{1 i}+\beta 22 x 2 i\right)
\end{gathered}
$$

where $i=1, \ldots, n(n=197) x_{1 i}$ denotes the age at diagnosis of diabetes (in years) and $x_{2 i}$ is a binary variable denoting the type of diabetes $(1=$ juvenile; $0=$ adult $)$.

We consider the same priors for the parameters, $\lambda_{1}, \lambda_{2}, \theta_{1}, \varphi_{11}, \varphi_{10}, \varphi_{01}$ and $\varphi_{00}$, and the same Gibbs sampling steps using the rjags library in $\mathrm{R}$ software used for the bivariate Weibull distribution derived from FGM copula in presence of cure fraction. For the others parameters we consider independent priors $\alpha_{l} \sim \operatorname{Gamma}(0.001,0.001), \beta_{l 1} \sim N(0,1000)$ and $\beta_{l 2} \sim N$ $(0,1000), l=1,2$, were $N(e, f)$ denotes a normal distribution with mean $e$ and variance $f$. In Table 3, we have the posterior summaries of interest assuming "FGM copula model" in the presence of cure fraction and the covariates age and type of diabetes.

From the results of Table 3, we observe that both covariates (age at diagnosis and type of diabetes) do not present significant effects on the response since the $95 \%$ credible intervals contain the zero value. In fact the Monte Carlo estimates of DIC are very similar considering the dependent model in presence or not of covariates. Considering the independent model, we observe a DIC value a little bit larger, an indication that the dependence model is better fitted by the data.

Table 3: Posterior summaries ("FGM copula model", considering cure fraction and covariates)

\begin{tabular}{ccccc}
\hline Parameter & Mean & S.D. & $95 \%$ Credible Interval & DIC \\
\hline$\alpha_{1}$ & 102.05 & 138.99 & $(6.20 ; 514.62)$ &
\end{tabular}




$\begin{array}{lcccc}\beta_{11} & 0.006 & 0.03 & (-0.05 ; 0.06) & \\ \beta_{12} & -0.66 & 0.78 & (-2.19 ; 0.88) & \\ \alpha_{2} & 68.48 & 40.83 & (20.21 ; 167.88) & \\ \beta_{21} & -0.02 & 0.01 & (-0.05 ; 0.008) & \\ \beta_{22} & -0.12 & 0.42 & (-0.97 ; 0.74) & \\ \lambda_{1} & 0.99 & 0.16 & (0.70 ; 1.32) & 1667.57 \\ \lambda_{2} & 0.93 & 0.09 & (0.77 ; 1.12) & \\ \theta_{1} & 0.01 & 0.59 & (-0.96 ; 0.95) & \\ \varphi 11 & 0.41 & 0.10 & (0.27 ; 0.67) & \\ \varphi 10 & 0.04 & 0.03 & (0.002 ; 0.12) & \\ \varphi 01 & 0.30 & 0.10 & (0.05 ; 0.47) & \\ \varphi 00 & 0.25 & 0.05 & (0.14 ; 0.36)\end{array}$

In this case, we also could to model the data considering other existing copula functions since the FGM copula is appropriated only to model weak dependence Nelsen (2006). Observe that the $95 \%$ credible interval of the dependence parameter $\theta_{1}$ includes the zero value, implying that $\theta_{1}$ is insignificant and it is enough to justify that $T_{1}$ and $T_{2}$ are not dependent at least through FGM copula function.

Now, let us assume a bivariate Weibull lifetime distribution derived from the Gumbel copula in presence of cure fraction presented in Section 3.2 with independent priors $\lambda_{l} \sim$ $\operatorname{Gamma}(0.001,0.001), \mu_{l} \sim \operatorname{Gamma}(0.001,0.001), l=1,2$, and $\theta_{2} \sim U(0,1)$. We also assume a Dirichlet prior given by (38) for $\varphi_{11}, \varphi_{10}, \varphi_{01}$ and $\varphi_{00}$ with hyperparameter values $w_{1}=w_{2}=w_{3}=$ $w_{4}=1$ (noninformative priors for the incidence parameters).

Considering the rjags library Plummer (2011) in R software R Development Core Team (2011), a single chain has been used considering the simulation for each parameter for 210,000 times with a burn sample size of 50,000 to eliminate the possible effect of the initial values. We choose the simulated values from 100 to 100 , to get approximated uncorrelated values which result in a final chain of size 2,000. Convergence of the Gibbs Sampling algorithm was monitored using standard graphical methods, as the trace plots of the simulated samples Brooks and Gelman (1998).

In Table 4, we have the posterior summaries of interest (Monte Carlo estimates of the posterior means for each parameter). We also have in Table 4, the Monte Carlo estimate of DIC (Deviance Information Criterion), introduced by Spiegelhalter et al. (2002), used as a discrimination criterion for different models.

Smaller values of DIC indicates better models. 
Table 4: Posterior summaries (“Gumbel copula model”, considering cure fraction)

\begin{tabular}{ccccc}
\hline Parameter & Mean & S.D. & $95 \%$ Credible Interval & DIC \\
\hline$\lambda_{1}$ & 0.85 & 0.13 & $(0.63 ; 1.13)$ & \\
$\lambda_{2}$ & 0.94 & 0.09 & $(0.77 ; 1.14)$ & \\
$\mu_{1}$ & 62.14 & 23.27 & $(22.61 ; 109.41)$ & \\
$\mu_{2}$ & 28.99 & 5.36 & $(20.92 ; 41.89)$ & \\
$\theta_{2}$ & 0.55 & 0.20 & $(0.16 ; 0.91)$ & 1662.86 \\
$\varphi 11$ & 0.50 & 0.10 & $(0.29 ; 0.67)$ & \\
$\varphi 10$ & 0.04 & 0.04 & $(0.001 ; 0.14)$ & \\
$\varphi 01$ & 0.14 & 0.12 & $(0.005 ; 0.41)$ & \\
$\varphi 00$ & 0.31 & 0.05 & $(0.21 ; 0.40)$ &
\end{tabular}

Finally, assuming "Gumbel copula model" in presence of cure fraction and covariates, we assumed the same regression model (43) and consider the same priors for the parameters, $\lambda_{1}, \lambda_{2}$, $\theta_{1}, \varphi_{11}, \varphi_{10}, \varphi_{01}$ and $\varphi_{00}$, and the same Gibbs sampling steps using the rjags library in R software used for the bivariate Weibull distribution derived from Gumbel copula in presence of cure fraction. For the others parameters we consider independent priors $\alpha_{l} \sim \operatorname{Gamma}(0.001,0.001)$, $\beta_{l 1} \sim N(0,1000)$ and $\beta_{l 2} \sim N(0,1000), l=1,2$. In Table 5, we have the posterior summaries of interest assuming "Gumbel copula model" in the presence of cure fraction and the covariates age and type of diabetes. From the results of Table 5, we also conclude that both the covariates do not have significant effects on the response, but the dependent model derived from Gumbel copula gives smaller values of DIC, possibly an indication of better fit of the model for the data.

Table 5: Posterior summaries ("Gumbel copula model", considering cure fraction and covariates)

\begin{tabular}{ccccc}
\hline Parameter & Mean & S.D. & $95 \%$ Credible Interval & DIC \\
\hline$\alpha_{1}$ & 130.17 & 136.89 & $(11.42 ; 505.52)$ & \\
$\beta_{11}$ & -0.0001 & 0.02 & $(-0.05 ; 0.05)$ & \\
$\beta_{12}$ & -0.85 & 0.70 & $(-2.24 ; 0.53)$ & \\
$\alpha_{2}$ & 60.81 & 37.19 & $(19.91 ; 156.39)$ & \\
$\beta_{21}$ & -0.02 & 0.01 & $(-0.05 ; 0.005)$ & \\
$\beta_{22}$ & -0.15 & 0.43 & $(-1.01 ; 0.67)$ & \\
$\lambda_{1}$ & 0.88 & 0.14 & $(0.64 ; 1.19)$ & 1661.68
\end{tabular}




$\begin{array}{cccc}\lambda_{2} & 0.93 & 0.08 & (0.77 ; 1.10) \\ \theta_{2} & 0.53 & 0.20 & (0.13 ; 0.90) \\ \varphi 11 & 0.49 & 0.10 & (0.31 ; 0.66) \\ \varphi 10 & 0.03 & 0.03 & (0.0005 ; 0.09) \\ \varphi 01 & 0.18 & 0.11 & (0.01 ; 0.40) \\ \varphi 00 & 0.31 & 0.05 & (0.21 ; 0.40)\end{array}$

Since, in this case, it is not possible to compare the Kaplan-Meier curves with the estimated survival functions, we could use other discrimination methods to choose the best model for the data set. A possibility to discriminate the proposed models in terms of better fit for the data not considering the presence of covariates is to compare the Monte Carlo estimates of the means for the lifetimes $T_{1}$ and $T_{2}$ using the bivariate Weibull distributions derived from copula functions. Standard non-parametric estimates for the means based on Kaplan-Meier estimates are given, respectively, by $E\left(T_{1}\right)=57.83$ and $E\left(T_{2}\right)=43.53$ (use of the R software). Using the bivariate Weibull distributions derived from copula functions in presence of cure fraction, we get estimates for the means of $T_{1}$ and $T_{2}$ from (37), given by:

- FGM copula in presence of cure fraction not considering the presence of covariates: $E$ $\left(T_{1}\right)=36.08$ and $E\left(T_{2}\right)=25.62$.

- Independent lifetimes with Weibull distributions in presence of cure fraction not considering the presence of covariates: $E\left(T_{1}\right)=22.42$ and $E\left(T_{2}\right)=23.87$.

- Gumbel copula in presence of cure fraction not considering the presence of covariates: $E\left(T_{1}\right)=36.87$ and $E\left(T_{2}\right)=19.28$.

From these results, we also observe that the dependent models based on the copula functions are better fitted by the data, as compared to the independent Weibull model, since the obtained Bayesian estimates using copulas for the means of the marginal lifetimes are more close to the Kaplan-Meier estimates.

\section{Concluding Remarks}

The use of copula functions could be a good alternative to analyse bivariate lifetime data in presence of censored data, cure fraction and covariates. Observe that in many applications of lifetime modeling we could have the presence of a cure fraction for individuals that are "long term survivors" or "cured individuals".

In this paper, we have used two copula functions, but we also could consider any other existing copula function to build new bivariate lifetime models. We also have used marginal Weibull distributions, since this distribution has a good flexibility of fit for lifetime data, as 
constant, increasing or decreasing hazard functions. Other usual existing univariate lifetime distributions could be used (Gamma, log-normal, exponential, exponentiated Weibull distributions, among many others) to model the marginal distributions. This approach could be of great interest in many areas of interest, especially in medical or engineering studies.

The use of standard existing MCMC methods, especially with the rjags library in $\mathrm{R}$ software, could give a great simplification to obtain inferences for the proposed models. Using a real data set example, we observed that using both proposed models (FGM or Gumbel copulas) give similar results when observing the Monte Carlo estimates for the posterior means and credible intervals for all parameters, except for the association parameters $\theta_{1}$ and $\theta_{2}$, which have different interpretations Nelsen (2006). A small improvement for the fit of the models for the data is observed considering "Gumbel copula model" where the DIC values are smaller than considering "FGM copula model" (see Tables 1-5). Observe that prior information on the association parameter of copula functions usually it is not available from preliminary studies.

It is interesting to observe that we have great difficulties to get standard classical inferences for the parameters of the proposed models. In our case, we tried to find maximum likelihood estimators for the parameters of the models in our application using SAS software, but we did not get good results. Other problem with the use of classical approach: confidence intervals and hypothesis tests are based on asymptotic results. In our case, these results could be not accurate even using large sample sizes.

It is important to point out that any existing parametric lifetime distribution could be considered for the lifetimes of the susceptible individuals by choosing different parametric forms for $f_{10}\left(t_{1}\right), f_{01}\left(t_{2}\right), S_{10}\left(t_{1}\right)$ and $S_{01}\left(t_{2}\right)$ used in Sections 3.1 and 3.2 in the analysis of lifetimes in presence of cure fraction and censored data.

\section{References}

[1] Arnold, B. C. and Strauss, D. (1988). Bivariate distributions with exponential conditionals. Journal of the American Statistical Association, 83(402):522-527.

[2] Berkson, J. and Gage, R. P. (1952). Survival curve for cancer patients following treatment. Journal of the American Statistical Association, 47:501-515.

[3] Block, H. W. and Basu, A. P. (1974). A continuous bivariate exponential extension. Journal of the American Statistical Association, 69:1031-1037.

[4] Boag, J. (1949). Maximum likelihood estimation of the proportion of patients cured by Cancer therapy. Journal of the Royal Statistical Society, B, 11:15-53.

[5] Brooks, S. P. and Gelman, A. (1998). General Methods for Monitoring Convergence of Iterative Simulations. Journal of Computational and Graphical Statistics, 7(4):434-455. 
[6] Cancho, V. G. and Bolfarine, H. (2001). Modeling the presence of immunes by using the exponentiated-Weibull model. Journal of Applied Statistics, 28(6):659- 671.

[7] Casella, G. and George, E. I. (1992). Explaining the Gibbs sampler. Amer. Statist., 46(3):167-174.

[8] Chatterjee, N. and Shih, J. (2001). A bivariate cure-mixture approach for modeling familial association in diseases. Biometrics, 57(3):779-786.

[9] Chatterjee, N. and Shih, J. H. (2003). On use of bivariate survival models with cure fraction. Biometrics, 59(4):1184-1185.

[10] Chib, S. and Greenberg, E. (1995). Undestanding the metropolis-hastings algorithm. The American Statistician, 49(4):327-335.

[11] Copas, J. B. and Heydari, F. (1997). Estimating the risk of reoffending by using exponential mixture models. Journal of the Royal Statistical Society, A, 160(2):237-252.

[12] De Angelis, R., Capocaccia, R., Hakulinen, T., Soderman, B., and Verdecchia, A. (1999). Mixture models for cancer survival analysis: application to populationbased data with covariates. Statistics in Medicine, 18(4):441-454.

[13] Downton, F. (1970). Bivariate exponential distributions in reliability theory. Journal of the Royal Statistical Society, Series B, Methodological, 32:408-417.

[14] Dunsmuir, W., Tweedie, R., Flack, L., and Mengersen, K. (1989). Modeling the transitions between employement states for young australians. Australian Journal of Statistics, 31(A):165196.

[15] Farewell, V. T. (1982). The use of mixture models for the analysis of survival data with longterm survivors. Biometrics, 38:1041-1046.

[16] Farewell, V. T. (1986). Mixture models in survival analysis: Are they worth the risk? The Canadian Journal of Statistics, 14(3):257-262.

[17] Freund, J. E. (1961). A bivariate extension of the exponential distribution. Journal of the American Statistical Association, 56:971-977.

[18] Gamel, J. W., McLean, I. W., and Rosenberg, S. H. (1990). Proportion cured and mean log survival time as functions of tumor size. Statistics in Medicine, 9:999-1006.

[19] Gamel, J. W., Mclean, I. W., and Rosenberg, S. H. (1999). Proportion cured and mean logsurvival time as functions of tumor size. Statistical in Medicine, 9:999-1006. 
[20] Gelfand, A. E. and Smith, A. F. M. (1990). Sampling-based approaches to calculating marginal densities. Journal of the American Statistical Association, 85(410):398-409.

[21] Ghitany, M. E. and Maller, R. A. (1992). Asymptotic results for exponential mixture models with long term survivors. Statistics, 23:321-336.

[22] Gumbel, E. J. (1960). Bivariate exponential distributions. Journal of the American Statistical Association, 55:698-707.

[23] Hawkes, A. G. (1972). A bivariate exponential distribution with applications to reliability. Journal of the Royal Statistical Society, Series B, Methodological, 34:129-131.

[24] Haybittle, J. L. (1965). A two parameter model for the survival curve of treated cancer patients. Journal of the American Statistical Association, 53:16-26.

[25] Hougaard, P. (1986). A class of multivariate failure time distributions. Biometrika, 73(3):671678.

[26] Huster, W. J., Brookmeyer, R., and Self, S. G. (1989). Modelling paired survival data with covariates. Biometrics, 45(1):145-156.

[27] Kannan, N., Kundu, D., Nair, P., and Tripathi, R. C. (2010). The generalized exponential cure rate model with covariates. Journal of Applied Statistics, 37(9-10):1625-1636.

[28] Lambert, P. C., Dickman, P. W., Weston, C. L., and Thompson, J. R. (2010). Estimating the cure fraction in population-based cancer studies by using finite mixture models. Journal of the Royal Statistical Society. Series C. Applied Statistics, 59(1):35-55.

[29] Lambert, P. C., Thompson, J. R., Weston, C. L., and Dickman, P. W. (2007). Estimating and modeling the cure fraction in population-based cancer survival analysis. Biostatistics, 8(3):576594.

[30] Lawless, J. F. (1982). Statistical models and methods for lifetime data. John Wiley and Sons, New York.

[31] Maller, R. A. and Zhou, X. (1996). Survival analysis with long-term survivors. Wiley Series in Probability and Statistics: Applied Probability and Statistics. John Wiley \& Sons Ltd., Chichester.

[32] Marshall, A. W. and Olkin, I. (1967). A multivariate exponential distribution. Journal of the American Statistical Association, 62:30-44.

[33] Meeker, W. Q. (1987). Limited failure population life tests: Application to integrated circuit reliability. Technometrics, 29(1):51-65. 
[34] Morgenstern, D. (1956). Einfache Beispiele zweidimensionaler Verteilungen. Mitteilungsbl. Math. Statist., 8:234-235.

[35] Nelsen, R. B. (2006). An introduction to copulas. Springer Series in Statistics. Springer, New York, second edition.

[36] Ng, S. K. and McLachlan, G. J. (1998). On modifications to the long-term survival mixture model in the presence of competing risks. Journal of Statistical Computation and Simulation, 61:77-96.

[37] Peng, Y. and Dear, K. B. G. (2000). A nonparametric mixture model for cure rate estimation. Biometrics, 56:237-243.

[38] Plummer, M. (2011). rjags: Bayesian graphical models using MCMC. R package version 3-3.

[39] R Development Core Team (2011). R: A Language and Environment for Statistical Computing. R Foundation for Statistical Computing, Vienna, Austria. ISBN 3-900051-07-0.

[40] Sarkar, S. K. (1987). A continuous bivariate exponential distribution. Journal of the American Statistical Association, 82(398):667-675.

[41] Sklar, A. (2010). Fonctions de r'epartition a $n$ dimensions et leurs marges (republication). Annales de l'Institut de Statistique de Paris, 54(1-2):3-6. With an introduction by Denis Bosq.

[42] Spiegelhalter, D., Best, N., Carlin, B., and van der Linde, A. (2002). Bayesian measures of model complexity and fit. Journal of the Royal Statistical Society, Series B, Methodological, 64:583-639.

[43] Sy, J. P. and Taylor, J. M. G. (2000). Estimation in a Cox proportional hazards cure model. Biometrics, 56:227-236.

[44] Taylor, J. M. G. (1995). Semiparametric estimation in failure time mixture models. Biometrics, 51:899-907.

[45] Trivedi, P. K. and Zimmer, D. M. (2005). Copula Modeling. New Publishers, New York.

[46] Trivedi, P. K. and Zimmer, D. M. (2006). Copula Modeling: An Introduction for Practitioners, volume 1 .

[47] Tsodikov, A. D., Ibrahim, J. G., and Yakovlev, A. Y. (2003). Estimating cure rates from survival data: an alternative to two-component mixture models. Journal of the American Statistical Association, 98(464):1063-1078. 
[48] Wienke, A., Lichtenstein, P., and Yashin, A. I. (2003). A bivariate frailty model with a cure fraction for modeling familial correlations in diseases. Biometrics, 59(4):1178-1183.

[49] Wienke, A., Locatelli, I., and Yashin, A. I. (2006). The modelling of a cure fraction in bivariate time-to-event data. Austrian Journal of Statistics, 35(1):67-76.

[50] Yakovlev, A. Y., Tsodikov, A. D., and Asselain, B. (1996). Stochastic models of tumor latency and their biostatistical applications. World Scientific, Singapore.

[51] Yamaguchi, K. (1992). Accelerated failure-time regression model with a regression model for the survining fraction: an application to the analysis of permanent employment in japan. Journal of the American Statistical Association, 87:284- 292.

[52] Yu, B., Tiwari, R. C., and Cronin, K. Z. (2004). Cure fraction estimation from the mixture cure models for grouped survival times. Statistics in Medicine, 23:1733-1747.

Received May 15, 2015; accepted November 1, 2015.

Em'ilio A. Coelho-Barros

Universidade Tecnol’ogica Federal do Paran’a, DAMAT/UTFPR 


\section{A rjags Codes}

Listing 1: Main code of the rjags library.

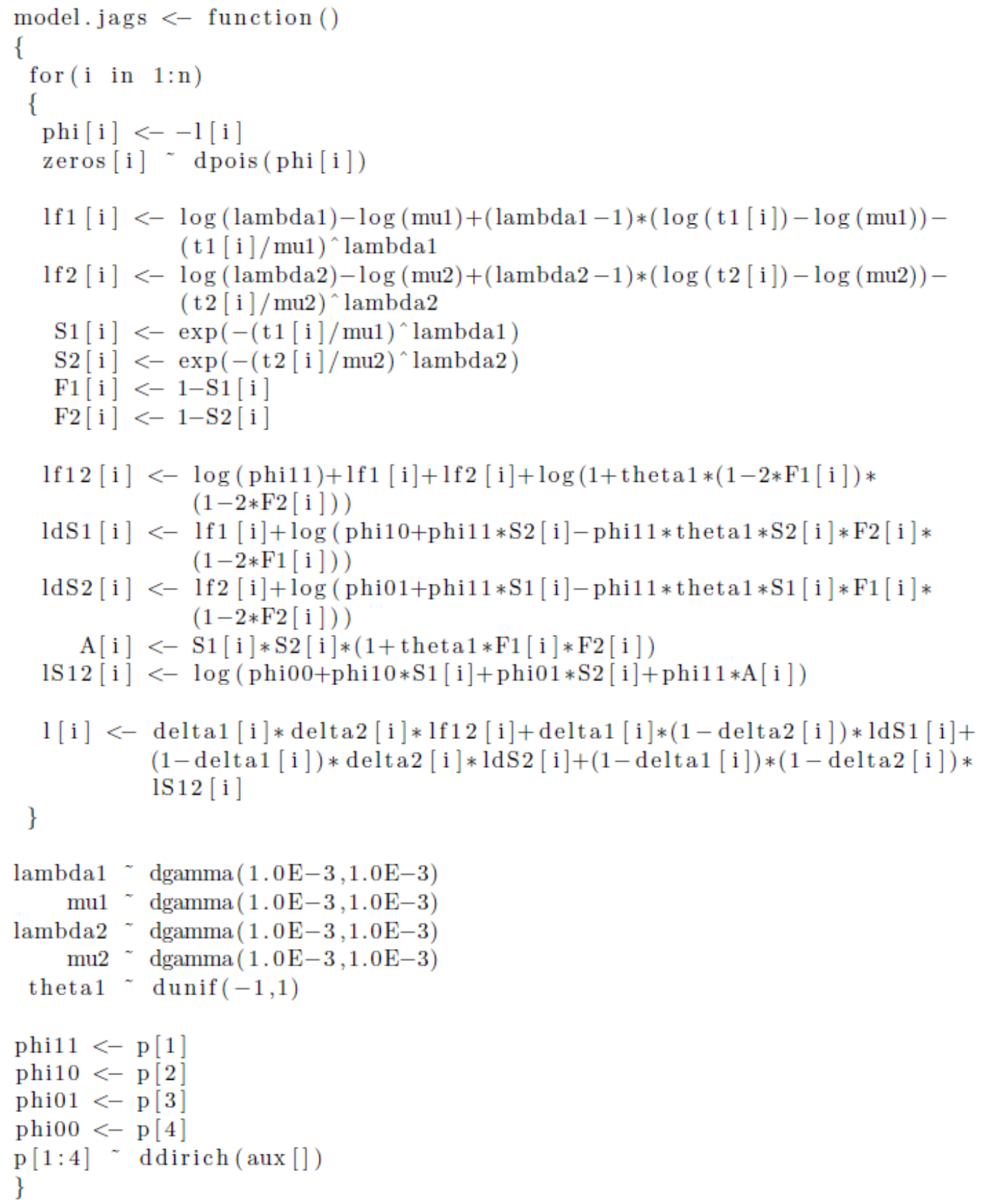

